\title{
Distributional Property of a Proportion Trait Whose Component Traits Follow a Normal Distribution
}

\author{
Masayuki SHOJO and Hiroaki IWAISAKI ${ }^{1}$ \\ Graduate School of Science and Technology, Niigata University, Niigata-shi 950-2181, Japan \\ ${ }^{1}$ Faculty of Agriculture, Niigata University, Niigata-shi 950-2181, Japan
}

(Received May 7, 1999 ; Accepted July 21, 1999)

\begin{abstract}
This paper concerns the characteristics of the distribution of a proportion trait that is defined as the proportion of the amount of one component to the total amount of all the components. Two component traits following curtailed normality are considered here and are assumed to have positive values. The probability density function for the proportion trait is derived in the general form and then the particular form is given for the case where coefficients of variation for the component traits are relatively small. Using three selected sets of the parameter values for the component traits, the parametric values of the location, dispersion, skewness and kurtosis for the distribution of the proportion trait are shown. It is revealed that the shape of the distribution is able to be symmetrical and very nearly normal, skewed to the right or skewed to the left, with a density more peaked around its center than a normal curve. These results confirm us that phenotypic selection differentials in one cycle of upward and downward truncation selection for the proportion trait may be asymmetrical, especially with higher intensity of selection, even when the component traits follow a normal distribution.
\end{abstract}

Animal Science Journal 70 (6) : 408-413, 1999

Key words : Proportion trait, Distribution, Probability density function, Moment, Curtailed normality

Traits defined as the proportion of the amount of one component to the total amount of all the components, or proportion traits, are sometimes of interest in animal and crop breeding applications. Proportion traits are classified as a particular type of ratio traits and the phenotypic values are distributed within the limited range. It has usually been assumed that a proportion trait is controlled indirectly by many genes which affect its component quantitative traits directly.

While some of distributional, statistical and genetic properties of the simplest ratio of the phenotypic values of two quantitative traits were studied $^{1-3,5,6)}$, for a proportion trait, Shojo and Iwaisaki $i^{9)}$ gave an expression for the truncation point, assuming truncation selection based on the proportion phenotypic values of individuals. They also examined the magnitudes of the discrepancy between the true value of and the normal approximation to the truncation point, given several fractions selected, and then suggested that the shape of the distribution of the proportion trait is changeable according to the parameter situations of the component traits.

The purpose of this paper is to verify the distributional property of a proportion trait, supposing that the component traits follow a curtailed normal distribution.

\section{Materials and Methods}

We denoted the phenotypic value of a proportion trait by

$$
r=x_{1} /\left(x_{1}+x_{2}\right),
$$

where $x_{j}(j=1,2)$ are the phenotypic values of the component traits with means $\mu_{\mathrm{j}}$, variances $\sigma_{\mathrm{j}}^{2}$, covariance $\sigma_{12}$ and correlation $\rho$. The phenotypic

Corresponding : Hiroaki IWAISAKI (fax : +81 (0) 25-262-6854, e-mail : iwsk@agr.niigata-u.ac.jp)

Anim. Sci. J. $70(6): 408-413,1999$ 
values $x_{j}$ were assumed to follow the bivariate curtailed normal distribution for which probability density function (pdf) is

$$
\begin{aligned}
f\left(x_{1}, x_{2}\right)= & {\left[.5 /\left\{\pi \sigma_{1} \sigma_{2}\left(1-\rho^{2}\right)^{1 / 2}\right\}\right] \exp [\{-.5 /} \\
& \left.\left(1-\rho^{2}\right)\right\}\left\{\left(\mathrm{x}_{1}-\mu_{1}\right)^{2} / \sigma_{1}^{2}-2 \rho\left(\mathrm{x}_{1}-\mu_{1}\right)\right. \\
& \left.\left.\left(\mathrm{x}_{2}-\mu_{2}\right) /\left(\sigma_{1} \sigma_{2}\right)+\left(\mathrm{x}_{2}-\mu_{2}\right)^{2} / \sigma_{2}^{2}\right\}\right] \\
& \left(0<\mathrm{x}_{\mathrm{j}}\right) .
\end{aligned}
$$

We first derived the pdf of $r$. As one expression, the pdf is written as

$$
f(r)=\int_{0}^{\infty} g\left(r, x_{2}\right) d x_{2},
$$

where $g\left(r, x_{2}\right)$ stands for the joint pdf of $r$ and $x_{2}$. Then, employing the transformation

$$
\mathrm{x}_{1}=\{\mathrm{r} /(1-\mathrm{r})\} \mathrm{x}_{2} \text { and } \mathrm{x}_{2}=\mathrm{x}_{2} \text {, }
$$

and consequently having the Jacobian $|\mathrm{J}|=\mid \mathrm{x}_{2} /$ (1 $-r)^{2} \mid$, we had the pdf of $r$ as

$$
f(r)=\int_{0}^{\infty}|J| f\left[\{r /(1-r)\}_{x_{2}}, x_{2}\right] d_{x_{2}} .
$$

Conducting the required integration, with some arrangement, we obtained

$$
\begin{aligned}
f(r)=h_{1}(r) \int_{L}^{\infty} t \phi(t) d t \\
\\
+h_{2}(r) \int_{L}^{\infty} \phi(t) d t,
\end{aligned}
$$

where $h_{1}(r), h_{2}(r)$ and $L$ are a function of $r$, and $\phi(t)$ is the pdf of the standardized normal variate, or

$$
\phi(t)=\left\{1 /(2 \pi)^{1 / 2}\right\} \exp \left(-.5 t^{2}\right) .
$$

Then, noticing the fact that $\sigma_{\mathrm{j}} / \mu_{\mathrm{j}} \rightarrow 0, \mathrm{~L} \rightarrow-\infty$, for the case where $\mathbf{L}$ is sufficiently negative to be replaced by $-\infty$, we had

$$
f(r) \approx h_{2}(r),
$$

assuming that $\int_{-\infty}^{\infty} t \phi(t) \mathrm{dt}=0$ and $\int_{-\infty}^{\infty} \phi(\mathrm{t}) \mathrm{dt}=1$.

For a comparison, we also dealt with the standard normal approximation to the pdf of $r\left(f_{A}(r)\right)$, which was written as

$$
\mathrm{f}_{\mathrm{A}}(\mathbf{r})=\left\{1 /(2 \pi \beta)^{1 / 2}\right\} \exp \left\{-.5(\mathbf{r}-\alpha)^{2} / \beta\right\},
$$

where

$$
\begin{gathered}
\alpha=\left\{\mu_{1}\left(\mu_{1}+\mu_{2}\right)^{2}+\mu_{1}\left(\sigma_{2}^{2}+\sigma_{12}\right)-\mu_{2}\left(\sigma_{1}^{2}+\sigma_{12}\right)\right\} / \\
\left(\mu_{1}+\mu_{2}\right)^{3}
\end{gathered}
$$

and

$$
\beta=\left(\mu_{2}^{2} \sigma_{1}^{2}-2 \mu_{1} \mu_{2} \sigma_{12}+\mu_{1}^{2} \sigma_{2}^{2}\right) /\left(\mu_{1}+\mu_{2}\right)^{4},
$$

being based on a Taylor-series expansion of $r$ around the means $\left(\mu_{1}, \mu_{2}\right)$ and dropping all terms of order higher than 2.

Next, to characterize the distribution of $r$ in detail, using the derived pdf and computing the 1 st raw moment $\left(m_{1}\right)$ and the 2 nd to 4 th central moments $\left(M_{k} ; k=2,3,4\right)$, we calculated mean $\left(P_{1}\right)$, variance $\left(P_{2}\right)$, coefficient of skewness $\left(P_{3}\right)$ and coefficient of kurtosis $\left(\mathrm{P}_{4}\right)$, as follows ${ }^{7)}$

$$
\begin{aligned}
& \mathbf{P}_{1}=\mathrm{m}_{1} \\
& \mathbf{P}_{2}=\mathbf{M}_{2} \\
& \mathbf{P}_{3}=\mathbf{M}_{3} / \mathbf{P}_{2}^{3 / 2} \\
& \mathbf{P}_{4}=\mathbf{M}_{4} / \mathbf{P}_{2}^{2}-3,
\end{aligned}
$$

and

where with denoting the appropriate region for integration by $R$,

$$
\mathrm{m}_{1}=\int_{R} \mathrm{rf}(\mathrm{r}) \mathrm{dr}
$$

and

$$
\mathbf{M}_{\mathrm{k}}=\int_{R}\left(\mathbf{r}-\mathbf{P}_{1}\right)^{\mathrm{k}} \mathbf{f}(\mathbf{r}) \mathrm{dr} .
$$

Numerical integrations were performed using IMSL routine DQDAG by a globally adaptive scheme based on the 30-61 points Gauss-Kronrod rule ${ }^{4)}$.

To confirm the validity of the derived pdf of $r$, on the other hand, Monte Carlo simulation was carried out. A population of two million of individuals was simulated, in which $\mathrm{x}_{\mathrm{j}}$ were generated, as follows

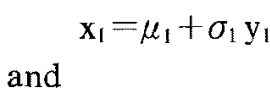

$$
\mathrm{x}_{2}=\mu_{2}+\sigma_{2}\left\{\rho \mathrm{y}_{1}+\left(1-\rho^{2}\right)^{1 / 2} \mathrm{y}_{2}\right\},
$$

where $y_{j}(j=1,2)$ represent two standard normal random variates, which were generated using the GASDEV function ${ }^{8}$. Then the phenotypic value of the proportion trait $(r)$ for each individual was formed as $\mathrm{x}_{1} /\left(\mathrm{x}_{1}+\mathrm{x}_{2}\right)$.

The generated data of $r$ were divided into 100 classes, and then relative frequency density was calculated as each relative frequency divided by the class interval. Moreover, for the distribution of the data, mean $\left(S_{1}\right)$, variance $\left(S_{2}\right)$, coefficient of skewness $\left(S_{3}\right)$ and coefficient of kurtosis $\left(\mathrm{S}_{4}\right)$ were also computed by the usual way, as follows

$$
\begin{aligned}
& \mathrm{S}_{\mathbf{1}}=\sum \mathrm{r} / \mathrm{n} \\
& \mathrm{S}_{2}=\sum\left(\mathrm{r}-\mathrm{S}_{1}\right)^{2} / \mathbf{n} \\
& \mathbf{S}_{3}=\sum\left(\mathrm{r}-\mathrm{S}_{1}\right)^{3} /\left(\mathrm{nS}_{2}^{3 / 2}\right)
\end{aligned}
$$

and

$$
\mathrm{S}_{4}=\sum\left(\mathrm{r}-\mathrm{S}_{1}\right)^{4} /\left(\mathrm{n} \mathrm{S}_{2}^{2}\right)-3,
$$

where $n$ is the number of individuals.

As assumed values of the parameters for the component traits, the three sets (Sets I, II and III) as considered by Shojo and Iwaisaki ${ }^{9)}$ were used. The values of the parameters $\left(\mu_{1}, \sigma_{1} / \mu_{1}, \mu_{2}, \sigma_{2} / \mu_{2}\right)$ were $(1.0, .05$, $1.0, .05),(1.0, .05,2.5, .2)$ and $(2.5, .2,1.0, .05)$ for Sets I, II and III, respectively, with assuming $\rho=.5$. 
In Monte Carlo simulation, 10 replicates were conducted for each of the sets, and the averages for $S_{k}$ were obtained.

\section{Results and Discussion}

The functions $h_{1}(r)$ and $h_{2}(r)$ in equation (5) were given as

$$
\begin{gathered}
\mathrm{h}_{1}(\mathrm{r})=\left[\left(1-\rho^{2}\right)^{1 / 2} /\left\{(2 \pi)^{1 / 2} \sigma_{1} \sigma_{2}(1-r)^{2} \mathrm{a}\right\}\right] \exp \\
\left\{-.5 \mathrm{~d} /\left(1-\rho^{2}\right)\right\}
\end{gathered}
$$

and

$$
\begin{gathered}
\mathbf{h}_{2}(\mathbf{r})=\left[\mathrm{b} /\left\{(2 \pi)^{1 / 2} \sigma_{1} \sigma_{2}(1-\mathbf{r})^{2} \mathrm{a}^{3 / 2}\right\}\right] \\
\exp \left\{-.5 \mathrm{~d} /\left(1-\rho^{2}\right)\right\},
\end{gathered}
$$

where

$$
\begin{aligned}
\mathrm{a}= & \mathbf{r}^{2} /\left\{\sigma_{1}^{2}(1-\mathbf{r})^{2}\right\}-2 \rho \mathbf{r} /\left\{\sigma_{1} \sigma_{2}(1-\mathbf{r})\right\}+1 \\
& / \sigma_{2}^{2}, \\
\mathrm{~b}= & \mu_{1} \mathbf{r} /\left\{\sigma_{1}^{2}(1-\mathbf{r})\right\}-\rho\left\{\mu_{1}(1-\mathbf{r})+\mu_{2} \mathbf{r}\right\} \\
& /\left\{\sigma_{1} \sigma_{2}(1-\mathbf{r})\right\}+\mu_{2} / \sigma_{2}^{2} \\
\mathrm{c}= & \mu_{1}^{2} / \sigma_{1}^{2}-2 \rho \mu_{1} \mu_{2} /\left(\sigma_{1} \sigma_{2}\right)+\mu_{2}^{2} / \sigma_{2}^{2}
\end{aligned}
$$

and

$$
d=c-b^{2} / a .
$$

The lower limit in the integration, $L$, was expressed as

$$
\mathrm{L}=-\mathrm{b} /\left\{\left(1-\rho^{2}\right) \mathrm{a}\right\}^{1 / 2} \text {. }
$$

The validity of the assumption put on $L$ to derive equation (6) from equation (5) was numerically examined for Sets I, II and III, considering the range of $r$ corresponding to that from -4.9 to 4.9 in the standard normal distribution (Table 1). The range was adopted as an enough range fulfilling the condition $\mathrm{x}_{\mathbf{j}}>0$ and was obtained based on the theory and the actual formula for the proportion truncation

Table 1. Ranges of $\mathrm{r}$ and $\mathrm{L}$ for the three sets ${ }^{1)}$

\begin{tabular}{crrrr}
\hline \hline Variable $^{2)}$ & Range $^{3)}$ & \multicolumn{1}{c}{ Set I } & \multicolumn{1}{c}{ Set II } & \multicolumn{1}{c}{ Set III } \\
\cline { 1 - 5 } r & Max & .5627 & .9461 & .8222 \\
& Min & .4373 & .1778 & .0539 \\
L & Max & -22.5682 & -20.2317 & -20.2317 \\
& Min & -23.0940 & -20.8167 & -20.8167 \\
\hline
\end{tabular}

1) Set I : $\mu_{1}=1.0, \sigma_{1} / \mu_{1}=.05, \mu_{2}=1.0$ and $\sigma_{2} / \mu_{2}=.05$; Set II : $\mu_{1}=1.0, \sigma_{1} / \mu_{1}=.05, \mu_{2}=2.5$ and $\sigma_{2} / \mu_{2}=.2$; Set III : $\mu_{1}=2.5, \sigma_{1} / \mu_{1}=.2, \mu_{2}=1.0$ and $\sigma_{2} / \mu_{2}=.05$. Correlation between $\mathrm{x}_{\mathrm{j}}, \rho$, is .5 for all the sets.

2) See text for notations.

3) Max : maximum value observed, Min : minimum value observed.
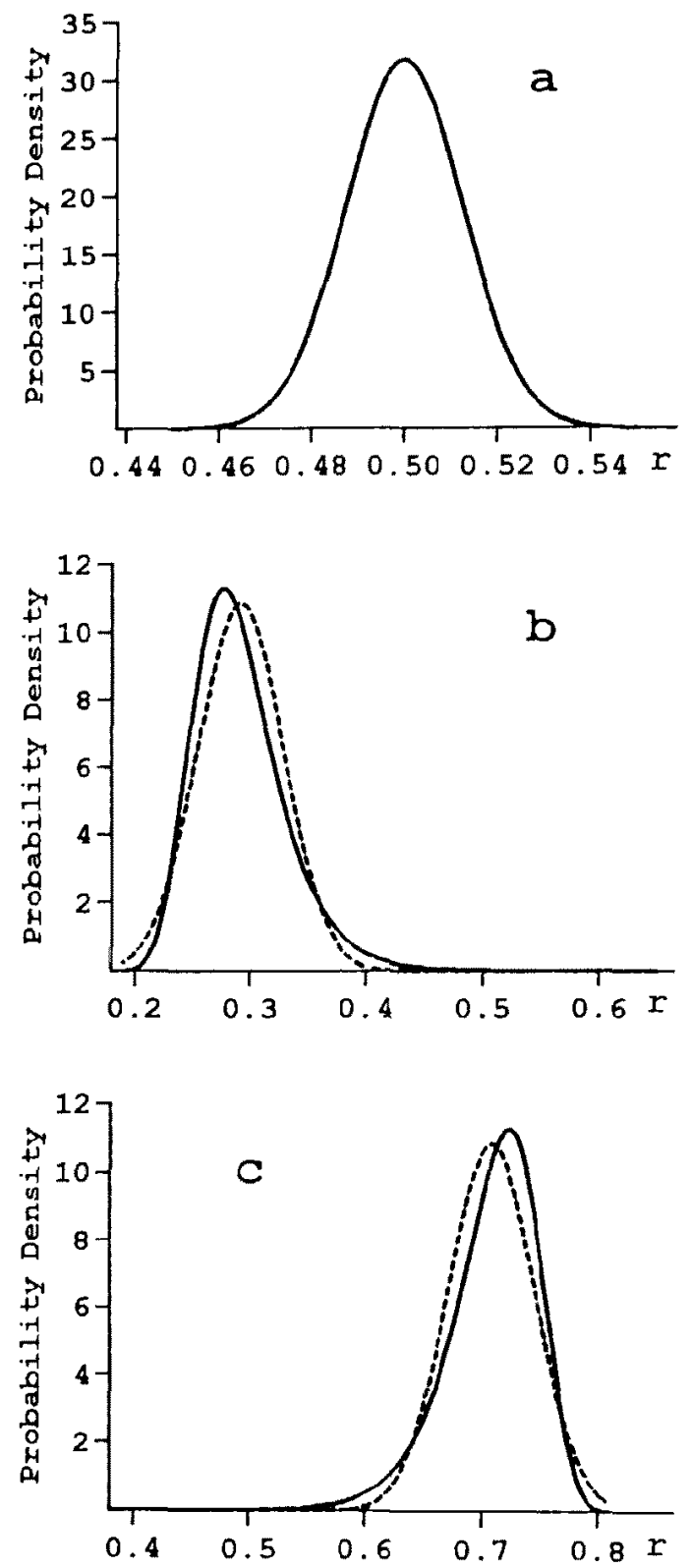

Fig. 1. Density functions of the r-distribution (solid line) and the normal approximation (dashed line) plotted for Sets I (a), II (b), and III (c).

point ${ }^{9)}$. Note that the probability integral for the given range results in 999999 . The maximum value of $\mathrm{L}$ was less than -20 for all the sets. This fact obviously shows that $\int_{\mathrm{L}}^{\infty} \mathrm{t} \phi(\mathrm{t}) \mathrm{dt}$ and $\int_{\mathrm{L}}^{\infty} \phi(\mathrm{t}) \mathrm{dt}$ are essentially 0 and 1 , respectively and consequently it is quite reasonable to replace $L$ by $-\infty$. For the cases where the magnitudes of coefficients of variation for $x_{j}$ are under $20 \%$, therefore, it is seen that equation (6) 
Distributional Property of a Proportion Trait

Table 2. Comparison of the density values between $f(r)$ and $f_{A}(r)$ for the three sets ${ }^{1)}$

\begin{tabular}{|c|c|c|c|c|c|c|c|c|}
\hline \multicolumn{3}{|c|}{ Set I } & \multicolumn{3}{|c|}{ Set II } & \multicolumn{3}{|c|}{ Set III } \\
\hline $\mathrm{r}$ & $f(r)$ & $f_{A}(r)$ & $\mathrm{r}$ & $f(r)$ & $\mathrm{f}_{\mathrm{A}}(\mathrm{r})$ & $\mathrm{r}$ & $f(r)$ & $\mathrm{f}_{\mathrm{A}}(\mathrm{r})$ \\
\hline 4492 & .0102 & .0084 & .2165 & .7632 & 1. 3957 & 6345 & 1.6033 & 1. 3957 \\
\hline 4594 & .1751 & .1628 & .2314 & 3. 0338 & 2.9196 & 6494 & 2.5590 & 2.9196 \\
\hline 4695 & 1.6655 & 1.6389 & .2463 & 6. 7742 & 5. 1836 & .6643 & 3.9714 & 5. 1836 \\
\hline 4797 & 8.5215 & 8.5292 & .2612 & 10.0814 & 7. 8109 & .6792 & 5.8960 & $\% .8109$ \\
\hline 4898 & 22. 9139 & 22. 9471 & .2761 & 11.2733 & 9.9896 & .6941 & 8.1873 & 9.9896 \\
\hline .5000 & 31.9154 & 31.9154 & .2910 & 10.3089 & 10.8434 & .7090 & 10.3089 & 10.8434 \\
\hline .5102 & 22.9139 & 22.9471 & .3059 & 8. 1873 & 9.9896 & .7239 & 11.2733 & 9.9896 \\
\hline 5203 & 8.5215 & 8.5292 & .3208 & 5. 8960 & 7.8109 & .7388 & 10.0814 & 7. 8109 \\
\hline 5305 & 1.6655 & 1.6389 & .3357 & 3.9714 & 5. 1836 & .7537 & 6.7742 & 5. 1836 \\
\hline 5406 & .1751 & .1628 & .3506 & 2. 5590 & 2.9196 & .7686 & 3.0338 & 2. 9196 \\
\hline 5508 & .0102 & .0084 & .3655 & 1. 6033 & 1. 3957 & .7835 & 7632 & 1. 3957 \\
\hline
\end{tabular}

"See Table 1 for the parameter values.

is exact enough to describe the actual appearance of the pdf of $r$. Fortunately, the coefficients of variation would generally be less than $20 \%$ for most of quantitative traits of our concern.

The shapes of the distribution of $r$ for Sets I, II and III, which were based on $f(r)$ given by equation (6), are presented in Fig. 1 (a, b and $\mathrm{c}$ ), respectively, being compared to those of the normal approximation $f_{A}(A)$ expressed by equation (7). These plots based on $\mathrm{f}(\mathrm{r})$ agreed completely with the corresponding ones of the relative frequency densities calculated from the data generated actually (not shown). As a numerical illustration, for $f(r)$ and $f_{A}(r)$, the actual values of the densities were calculated for equally spaced, eleven selected values of $\mathrm{r}$ (Table 2), which well demonstrate the discrepancies between the true densities and the normal approximations. For Set I, the true distribution of $r$ was symmetrical, but except for the case where $r=.5$, the true densities were very slightly different from the normal approximations. For Sets II and III, note that the true densities in the upper tail for Set II correspond to those in the lower tail for Set III and vice versa, but $\mathrm{f}_{\mathrm{A}}(\mathrm{r}) \mathrm{s}$ for the two sets are the same, since $f_{A}(r)$ is simply based on use of the Taylorserics expansion. Also, we notice that even if $0<\mathrm{r}<$ 1 by definition, $r$ is totally distributed within a limited range in the cases of the lower coefficients of variation for $\mathrm{x}_{\mathrm{j}}$.

The parametric values of location, dispersion, skewness and kurtosis for the distribution of $r$, which were determined using equation (6), are shown in Table 3 for the three sets, together with the values calculated from Monte Carlo replicates. The values determined theoretically agreed very well with the corresponding values realized actually.

In the case of equal coefficients of variation for $x_{j}$ (Set $\mathbf{I}$ ), mean of $\mathrm{r}$ is essentially equal to $\mu_{1} /\left(\mu_{1}+\mu_{2}\right)$. With the unequal coefficients, however, it does not hold true. It has been shown that for Sets II and III, expected value of $\mathbf{r}$ is higher and lower than $\mu_{1} /\left(\mu_{1}+\right.$ $\left.\mu_{2}\right)$, respectively. The variance of $\mathbf{r}$ was found to be .0002 for Set $I$, and the values of this dispersion parameter for Sets II and III were the same, or .0016. Taking the precision of the numerical integration conducted into consideration, coefficient of skewness of the distribution of $\mathrm{r}$ was essentially zero for Set $\mathrm{I}$, while the values for Sets II and III were obviously different from the value expected for a symmetrical distribution, and it is quite likely that the values for the two sets are equal in absolute value. For kurtosis of the distribution, the value for Set II was equal to that for Set III and definitely higher than zero, indicating that a density is more peaked around its center than the density of a normal curve. Once 
SHOJO and IWAISAKI

Table 3. Some characteristics of the distribution of $\mathrm{r}$ for the three sets ${ }^{1}$

\begin{tabular}{lllll}
\hline \hline Measure & & \multicolumn{1}{c}{ Set I } & \multicolumn{1}{c}{ Set II } & \multicolumn{1}{c}{ Set III } \\
\hline Mean & $\mathrm{P}_{1}$ & .5000 & .2914 & .7086 \\
& $\mathrm{~S}_{1}$ & .5000 & .2914 & .7086 \\
Variance & $\mathrm{P}_{2}$ & .0002 & .0016 & .0016 \\
& $\mathrm{~S}_{2}$ & .0002 & .0016 & .0016 \\
Skewness & $\mathrm{P}_{3}$ & .0001 & 1.0652 & -1.0651 \\
& $\mathrm{~S}_{3}$ & $-.0004(.0005)$ & $1.0651(.0017)$ & $-1.0648(.0020)$ \\
Kurtosis & $\mathrm{P}_{4}$ & .0224 & 2.6353 & 2.6352 \\
& $\mathrm{~S}_{4}$ & $.0228(.0005)$ & $2.6573(.0217)$ & $2.6354(.0210)$ \\
\hline
\end{tabular}

1) See Table 1 for the parameter values.

2) $\mathbf{P}_{\mathrm{K}}$ : determined using equation (6); $S_{K}$ : average calculated from 10 Monte Carlo replicates. Standard errors of $S_{1}$ and $S_{2}$ are all lower than $7 \times 10^{-6}$ and those of $S_{3}$ and $S_{4}$ are in parentheses.

again, for the $\mathrm{P}_{4}$ values concerning Sets II and III, the very slight difference in four decimal places would be due to the intrinsic precision of the numerical integration procedure used.

Assuming truncation selection based on the $\mathrm{r}$ values of individuals, Shojo and Iwaisaki $i^{9)}$ derived an appropriate expression for truncation point, given a fraction selected, in terms of the parameters for the component traits, numerically examined the magnitudes of the discrepancy between the exact point of truncation and its normal approximation for several fractions selected, and then indicated some possible deviations of the distribution of $r$ from normality. This study has revealed the property of the distribution of $r$ in details and brings a complete support to the indication by Shojo and Iwaisaki $i^{9)}$. It has been clearly shown that the shape of the distribution of the proportion trait is able to be symmetrical and very nearly normal, skewed to the right, or skewed to the left, even when the component traits follow (curtailed) normality, although the deviation from normality is likely to be not large in the cases where coefficients of variation for the component traits are relatively low. For one cycle of upward and downward truncation selection for the proportion trait, Shojo and Iwaisaki ${ }^{10)}$ studied expected selection differentials using the selection index method and the actually realized ones in a large simulated population, and reported that the selection differentials in the two directions were symmetrical for Set I, free of fractions selected, and asymmetrical for Sets II and III, especially with higher intensity of selection. The distributional property of the proportion trait as revealed in this paper is the reason why such phenomena occur.

\section{References}

1) Gunsett FC. Linear index selection to improve traits defined as ratios. Journal of Animal Science, 59 : 1185-1193. 1984.

2) Gunsett FC. Problems associated with selection for traits defined as a ratio of two component traits. Proceedings of the Third World Congress on Genetics Applied to Livestock Production, XI : 437-442. 1986.

3) Gunsett FC. Merit of utilizing the heritability of a ratio to predict the genetic change of a ratio. Journal of Animal Science, 65 : 936-942. 1987.

4) IMSL. IMSI, Math/Library Vols. 1 and 2. 591-594. Visual Numerics Inc. Houston. 1997.

5) Iwaisaki H, Wilton JW. Regression of genotypic on phenotypic value of a ratio-defined character. Biometrics, 49 : 1154-1163. 1993.

6) Mather DE, Gunsett FC, Allen OB, Kannenberg LW. Estimation of phenotypic selection differentials for prodicting genetic responses to ratio-based selection. Genome, $30: 838-843.1988$.

7) Mood AM, Graybill FA, Boes DC. Introduction to the Theory of Statistics. 3rd ed. 72-77. McGrawHill. Singapore. 1974. 
8) Press WH, Teukolsky SA, Vetterling WT, Flannery BP. Numerical Recipes in Fortran 77. 2nd ed. 279280. Cambridge Univ. Press. Cambridge. 1996.

9) Shojo M, Iwaisaki $\mathbf{H}$. Truncation point in truncation selection for a proportion-defined trait. Animal Science and Technology, $69: 417-423.1998$.
10) Shojo M, Iwaisaki H. Prediction of selection differentials expected from proportion-based truncation selection. Journal of Genetics and Breeding, 53 : 1999. (in press) 